Administrative Issues Journal: Connecting Education, Practice, and Research, Winter 2017, Vol. 7, No. 2: 57-69. DOI: 10.5929/2017.7.2.6

\title{
Migrating learning management systems: A case of a large public university
}

\author{
Brenda L. R. Such, Ph.D. \\ University of Florida \\ Albert D. Ritzhaupt, Ph.D. \\ University of Florida \\ George S. Thompson, Ed.S. \\ University of Florida
}

\begin{abstract}
In the past 20 years, institutions of higher education have made major investments in Learning Management Systems (LMSS). As institutions have integrated the LMS into campus culture, the potential of migrating to not only an upgraded version of the LMS, but also an entirely different $L M S$, has become a reality. This qualitative research study examines the perspectives of five stakeholders involved with the migration of an LMS at a major research institution in the southeastern United States. Using Lewin's (1947) Change Management Model and Enterprise Resource Planning (ERP) Model as analogies, this research seeks to understand the role and responsibilities of the various stakeholders, their decision-making, and the implications of the decisions on the migration process. Using Glaser and Strauss's (1967) constant comparative method and Charmaz's (2006) work related to grounded theory, four major categories emerged from our data: time as a catalyst for change, power of communication, compatibility of technologies, and faith in the system. The categories contribute to a preliminary model that may assist other institutions as they consider whether to migrate LMSs.
\end{abstract}

Keywords: learning management system, higher education, infrastructure, technology, decisionmaking, qualitative

ne of the most significant information and communication technology investments by institutions of higher education in the past decade has been the Learning Management System (LMS) (Coates, James, \& Baldwin, 2005; McGill \& Klobas, 2009). An LMS has as many definitions as there are software options, but, for the purpose of this paper, an LMS is an information system that facilitates learning, assessment, and other educational administrative functions for the organizations that integrate them into the fabric of their enterprises. An LMS stores, processes, and disseminates educational materials and supports administration and communication associated with teaching and learning (McGill \& Klobas, 2009).

SUCH, RITZHAUPT, \& THOMPSON / DOI: 10.5929/2017.7.2.6 
As academic institutions have integrated the LMS into their campus enterprise and culture, the possibility of a move to not only an upgraded version of the existing LMS, but also an entirely different LMS, has become an eventual point of consideration. Although unavoidable, academic institutions are hesitant to consider an upgraded or different LMS due to the finances, time, and effort that have already been invested in the integration of the initial LMS as a functioning component within the campus information system. A complicated but necessary web of the decision-making process includes key players such as administrators, faculty and staff members, students, instructional designers, and technical support. Furthermore, the potential change to a different LMS may expose risks for more financial expenditures, problems related to technological usability and integration, the daunting demand for quick pedagogical redevelopment of course curricula, and the unknown.

For the purposes of the study, we decided to use the term migration according to its definition as a "movement of people to a new area or country in order to find work or better living conditions" (Oxford Dictionaries Online). Although there is no physical movement per se in the design of the study, we determined that the move to a new LMS would be a migration-a movement in which the stakeholders at the university level are finding better conditions for academic purposes and praxis. We then used the term throughout the data collection and analysis processes to evaluate the reactions from the stakeholders and to gauge how the definition compared to the interviewed stakeholders' experiences.

\section{Conceptual Framework}

From a business perspective, we used the Lewin's (1947) Change Management Model and the Enterprise Resource Planning (ERP) Model. The former model provides an initial outlook of how people have managed change in an organizational setting, and the latter model breaks down an ERP system into key components.

\section{Lewin's Change Management Model}

Lewin's Change Management Model conveys that an organization undergoing change will realize that whatever has been solidified in the work system will be unfrozen. The intended change is then executed until the process is complete and refrozen. The last stage includes an affective component, which assures the involved persons that the change will remain and that the process will not occur again, or again soon. Within the context of migrating from one LMS to another, Lewin's Change Management Model assists both theory and practice in understanding how a new system is adopted and integrated into an organization. That is, the procedures and processes used to move from one LMS to the next are akin to this model for change.

\section{Enterprise Resource Planning Model}

Enterprise Resource Planning (ERP) software allows people to look at the breakdown of functions within an enterprise and to plan to maximize those functions (Hasselbring, 2000; Kremers \& Van Dissel, 2000; Leon, 2008). An ERP can facilitate the various functions of an entire organization such as financial management, supply chain management, manufacturing resource planning, human resource management, and customer relationship management.

SUCH, RITZHAUPT, \& THOMPSON / DOI: 10.5929/2017.7.2.6 
Based on the ERP model, we then constructed a tentative model to describe how the LMS is more than technology, but central to an academic institution as an enterprise. To better understand the effect of the LMS within the system, we first divided the system into key components, including accreditation, teaching, communication, learning, and assessment. We collected and analyzed the data with the mindset that a change in LMS had the potential to maximize the functions of each component. Figure 1 visualizes the reconceptualized model.

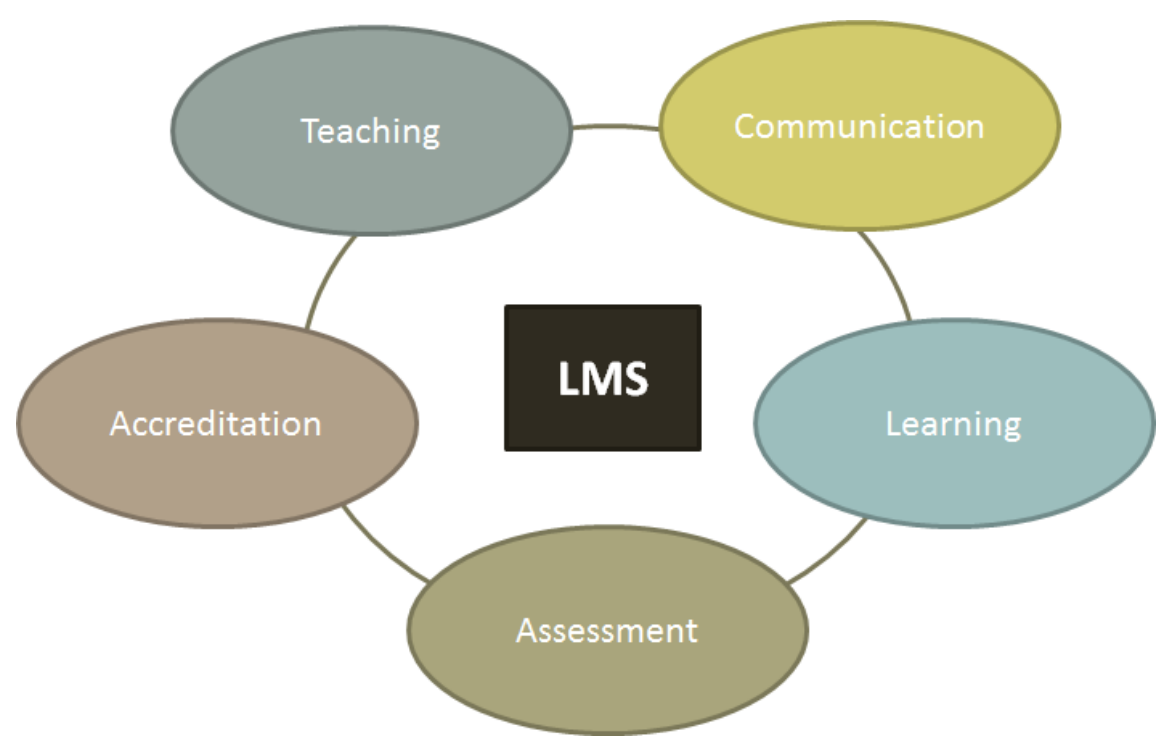

Figure 1. LMS within a comprehensive information system. This model considers the LMS not as an isolated technological system, but the center and functional influence of a larger system consisting of communication, learning, assessment, accreditation, and teaching.

To reiterate, the term "LMS" here is not merely the technology, but also the entire system in which the persons (e.g. students, faculty, and administration), the communicative and decision-making processes, and the technology are integral components. Additionally, the relationships among these components feed the functions of the LMS. We derived the following research questions based on this framework:

1. In the migration to a different LMS, what roles do responsible members within an academic enterprise hold? What decisions do they make?

2. What implications do those roles and decisions have in the migration process itself? 


\section{Supporting Literature}

When contemplating LMS change, it is helpful to consider historical factors responsible for the selection of existing LMSs. Selection and adoption by educational institutions have historically been vendor driven - the choice of LMS tools, options and functionality being predominantly determined by economic, usability, and vendor design factors, with less consideration to factors impacting teaching and learning (Sinkinson, Werner, \& Sieber, 2014). An LMS's usability and design are impacting factors in its utilization (Sturgess \& Nouwens, 2004). McGill and Klobas (2009) suggest that task-technology fit has a strong positive influence on student attitudes toward a perceived impact of an LMS on learning and has a marginal perceived positive influence on student grades. Simply put, an intuitive, easy-to-use interface contributes to LMS use and to positive student and instructor attitudes. Its uncluttered design fosters engagement and is conducive to learning, removing difficulties with the internal LMS navigation that could detract from learning. The concept of "form follows function" may concisely describe the most effective design and use of an LMS (Sullivan, 1896). Unfortunately, there is only a modest amount of extant research literature on criteria to be considered or the deliberative processes involved in selecting an LMS (Phelps \& Michea, 2003; Ray, 2009).

In addition to the influence of an LMS's usability and design on students' motivations for learning, aspects of an educational institution's culture can also affect the educational effectiveness of an LMS. These may include the use (or absence of) of research-based teaching methodology and strategies used to support instructors. The institutional LMS cannot be isolated from the larger institutional environment in which it resides. Therefore, institutional mores, particularly those exhibited by instructors concerning the LMS, also affect LMS efficacy by impacting student perceptions and motivations for learning (McGill \& Klobas, 2009). This inseparable relationship between the LMS and the institution necessitates that the LMS selection must consider the principles of good teaching and learning practice (Chickering \& Gamson, 1987; Harrington, Staffo, \& Wright, 2006). This is crucial since instructor and administrative attitudes can affect student learning (Liaw, 2008). Faculty and student attitudes may also affect perceived student and instructor efficacy and utilization of an LMS and any subsequent change and migrations of an LMS.

According to Beatty and Ulasewicz (2006), three primary institutional perceptions for LMS adoption are

1. The nature of LMSs implies a vehicle for increasing the efficiency of teaching.

2. LMSs are linked to prospects of enhanced student learning and recruitment.

3. LMSs help to meet student expectations and to assist an institution in its competition with other institutions.

Administrative reasons for LMS adoption also likely include the perceived control of teaching, learning, and administrative functions. These administrative pressures are likely responsible for the historical rapid development and the focus of an LMS on teaching, learning, and administration (Coates, James \& Baldwin, 2005). When considering LMS adoption and subsequent migration, institutions must include the realization that technologies will continue to evolve, and that infrastructure and policy modifications are necessary to effectively support the pursuit of innovation and relevancy.

SUCH, RITZHAUPT, \& THOMPSON / DOI: 10.5929/2017.7.2.6 


\section{Reasons for LMS Migration}

The selection of an LMS and subsequent migration should be overwhelmingly focused on the fact that LMSs are first and foremost tools for teaching and learning, and it is crucial that considerations of an LMS are informed and driven by pedagogical considerations. Otherwise, an LMS may be perceived by students as merely a convenient vehicle to retrieve course documents and to communicate with other instructors, hardly justifying the expense and potential disruption of moving to another system (Lonn \& Teasley, 2009).

Educational value is not determined solely by features and capabilities; value is obtained by LMS acceptance and use (Sturgess \& Nouwens, 2004; Coates et al., 2005). Reasons and motivations that institutions may wish to consider when migrating their systems include added functionality, compliance with new standards, expiration of support for installed version, keeping the LMS up-to-date, dissatisfaction with technical performance, organizational issues, and competitive pressures (Kremers \& Dissel, 2000). In addition, factors of economics and budget, along with the nature of technological systems that evolve constantly, may drive the will to change an LMS. LMS migration may be necessary simply because the hardware has become obsolete or too expensive to maintain, particularly given the development of cloud-based platforms (Ganesan, 2013; Jackson, 2014). In fact, "[the decision] whether to move to a new version or to a new product is not the issue because change is inherent in the use of technology" (Ryan, Toye, Charron, \& Park, 2012, p. 223). Technology change management is one of the main concerns of Information Technology departments, and institutions must continue to keep pace with technological innovation as much as possible to stay relevant and to simply survive (Mohawk College, 2009; Ryan et al., 2012). This educational technology arms race can be mitigated somewhat by thoughtful consideration and stakeholder involvement, using general lessons learned from the data migration from legacy databases to modern platforms in arenas other than educational ones (e.g. business and government).

\section{Issues with LMS Migration}

Many LMS platforms and products vary in their conformity to standards (Coates et al., 2005), and LMS vendors are highly motivated to emphasize proprietary features rather than support common standards in a protection of interest (Sinkinson et al., 2014). However, if the implementation of a new improved version of an LMS by the same vendor is the migration target, then migration might be more efficient due to vendor interest in customer retention, ease of transition due to similarity of platforms and increased sales for the vendor by marketing an institution's LMS of choice as a beneficial choice to other institutions (Kremers \& Dissel, 2000; Smart \& Meyer, 2005).

Most importantly, the consideration of LMS migration must include instructional design (instructor support services) and faculty advisory committees along with student input since instructors and students are ultimately the final arbiters of LMS effectiveness (Beatty \& Ulasewicz, 2006). There will arise, of course, faculty concerns and issues from an LMS change, including concerns about time demands and associated compensation, the possibility of instructor re-training, course re-design, assurances of knowledgeable support, copyright issues, communication issues, and a multitude of other concerns that could impact faculty and students attitudes and, therefore, the ultimate success of an LMS migration 
(Beatty \& Ulasewicz, 2006; Harrington et al., 2006; Kremers \& Dissel, 2000; Smart \& Meyer, 2005; Ryan et al., 2012; Turner, 2011).

To summarize, the successful changing of an institutional LMS and a migration from one LMS to another can be greatly facilitated by a planned iterative process, the involvement of all stakeholders (particularly faculty), and ample communication among all stakeholders during each step of the migration process.

\section{Data Collection}

We conducted qualitative research regarding the experience of a large research institution in the southeastern region of the United States as it migrated from the use of the LMS Blackboard to the LMS Canvas. (More about the timeline of the migration will be explained as a highlighted category from the data analysis.) As an academic enterprise, the university, established in the 1950s, is a high-impact, global research university that is one of the largest public universities in the nation and among the top 50 universities, public or private, for federal research expenditures. More than 47,000 students are enrolled, and the university offers nearly 250 degree programs at the undergraduate, graduate, specialty and doctoral levels. The university had been using Blackboard as the primary LMS for the institution for the past decade, and the migration to Canvas involved a combination of the parallel use of both LMSs and pilots with Canvas.

Before collecting data, we determined a tentative list of people to interview based on criteria of previous studies that indicated key personnel, including technology administrators, lead faculty members, and students (Harrington et al., 2006; Ryan et al., 2012; Smart \& Meyer, 2005). Then, using the list as a guide, we employed snowball sampling to determine who the persons being interviewed thought were key members in the migration process. Balancing against preconceived notions of the LMS migratory process (one team member had participated in an LMS migration as a faculty member and the other as an instructional designer), the unstructured interviews captured what the persons being interviewed considered as important to their own role and the migration process itself. Each interview lasted no longer than one hour, and a third-party agency transcribed the interviews for analysis. Because the study focused on the roles and the actions of the individuals interviewed, the following information provides the interviewed individuals' titles with their pseudonyms and quotes exhibiting the essence of their thoughts regarding the LMS migration.

\section{Voices within the Academic Enterprise}

Mitchell Sanderson, LMS Administrator. In regard to the initial thought of change: "Yeah, we don't call it migrating as much as we call it you know moving to, you know transitioning to a different system. But that's the same thing.... We had to make, any time we'd make a move to make a positive move towards improving the overall LMS, and not just make a lateral move because somebody wants to have a nonBlackboard environment."

Paul James, Director of Center for Faculty Support. In regard to the process of choosing an LMS: "I do think it was a mistake not to involve more faculty at a wider level." In regard to faculty members' transferring their courses to the new LMS: "But I'm not in control of that. So, you know, I have no under the hood access here. I can ring the bell and say, 'You know, Armageddon is coming. Back up your courses.'” 
Bill Langston, Associate Dean. In regard to faculty members transitioning to the new LMS: "Our faculty, you know, they let us know that they're displeased with the process, but they'll all be ready."

Jane Lewis, faculty member and representative on LMS selection committee. In regard to why she participated in the LMS migration: "I just can't live in two worlds, so I just figured I'm just going to start living in this place where I know we're going to be and just make it work."

Ruth Walters, Instructional Designer. In regard to facilitating the LMS migration as an instructional designer: "A lot of it just has to do with kind of knowing and understanding the mentality of faculty. You know, what they're willing to do themselves, and what they'd rather have someone else do.... Some you know, are very kind of off the cuff and really don't use the LMS much at all. It's kind of just a storage area. And then others exploit it more. What really helped in terms of transitioning to Canvas is that since I knew kind of their likes, dislikes, preferences, approaches, I could go into Canvas and say, 'Well, if you did this in Blackboard, you'd want use this tool in Canvas 'cause it's very similar.' Because like it or not, now that Blackboard is going away, it's like everybody loves it. You know, 'Oh my gosh, Blackboard, I loved it. I had everything in there, and everything works so perfectly.' Now that it's going away."

\section{Data Analysis and Findings}

Based on Glaser and Strauss's (1967) constant comparative method and Charmaz's (2006) work related to grounded theory, the data analysis was an iterative process in which the transcripts underwent multiple levels of coding (open, axial, and selective) and the codes were compared to one another for a proposed theoretical sampling. Coding was done by hand, and the creation and organization of a private research website facilitated the higher level of selective coding in which category development and themes were further scrutinized. Throughout the coding process, the codes were compared to the study's conceptual framework to further the discussion and provide possible implications about LMS migrations. The following are categories that are indicative of the properties and dimensions of the LMS migration seen in this study (Strauss \& Corbin, 1990).

\section{Time as a Catalyst for Change}

The factor of time contributed due to the fact that, at the time of discussions, the research institute's contract with Blackboard would terminate within two and a half years if not renewed. A small group, starting with 40 appointed members, began to look at LMS options during the summer semester, which included Angel, Canvas, Moodle, and an updated version of Blackboard. Having a strict deadline, the decision-makers realized that the entire institution would need to be prepared for an LMS change; based on the interviews, even choosing an upgraded version for the already employed Blackboard would require extensive preparation.

The time frame required an LMS to be chosen within three months, at which time Canvas was chosen. Volunteers began piloting courses in Canvas in the fall, and the entire following year was spent communicating with faculty members that their course materials were to be transferred from Blackboard to Canvas, although it was not until summer that there was more initiative to reach out to faculty members. Due to the recommendation that creating a course from scratch in the new LMS would be less 
frustrating than transferring course materials from Blackboard, training sessions and workshops for Canvas were set to occur twice a week, then more frequently. By leveraging the cut-off deadline, faculty members were more willing and steadily participated in the migratory process; however, within a year of the deadline, a large number of faculty members had not requested help from the center for faculty support. Although time seems to be an obvious factor in any decision-making process, the interviewees repeatedly mentioned that the reminders of a deadline were a catalyst for change.

\section{Power of Communication}

Throughout the interviews, the need for communication was also repeatedly emphasized. There was a lack of communication and transparency about the group that chose the LMS; some had said the group had appointed members while another reported that the group was composed of volunteers. Once the LMS was chosen, it was reported that it took about 10 months before faculty members were proactively reached.

The means of communication about the migration and trainings included a banner on the faculty development website and emails to the departments to disseminate to faculty members. Sharing information from one person to another had been effectual. Faculty members who had been trained went back to their departments and shared what they learned. In return, more faculty members from those departments began the process to transfer their courses. Rather than one person undergoing the migration process alone, it seemed more likely that people would change as a group. One instructor reported that she felt more comfortable with the transition process after she talked to her colleagues at other academic institutions that had experienced transitioning to a new LMS.

Because the research institute had multiple technology departments participating in the migration, each department had the same goal to facilitate the change but different responsibilities due to funding. The miscommunication among these departments about their responsibilities led to faculty runaround and frustration. Thus, in affirmation of the literature, communication (or the lack of it) among all stakeholders throughout the process was found to be a crucial factor affecting successful LMS migration.

\section{Compatibility of Technologies}

Despite the LMS being housed in technology, the compatibility of technologies at multiple levels was not a major concern. The literature confirmed this, since a properly planned migration would address and minimize potential compatibility issues and provide for response mechanisms if compatibility issues were to arise. An indispensable requirement for the selected LMS was integration with the institute's student information system and the tools that the institute had already created. However, other technological applications such as in-class recordings and the transferability of already recorded and edited class videos were not considered in the LMS decision-making process. The lack of consideration for the compatibility of these technologies with Canvas increased the stress of faculty members and the workload for instructional designers and the technology support departments.

\section{"Faith" in the System}

Although some may argue that migration may not be the best term to use for the process of moving from one LMS to another, the accounts of the interviewed decision-makers portrayed how the entire institution 
would be venturing into a new environment. The inevitable reality required faculty members, as well as adjuncts and teaching assistants, to build and teach new courses, the students to use a new interface, and technical and instructional support teams to learn a new system so well they could answer potential questions from faculty members and students. However, since much of the existing data (student and instructor records, course content, etc.) already existed in the previous LMS, migration is an apt term for describing the process of moving from one LMS to another.

Concerning the whole migratory process, the underlying tone was that decisions were made, but there was little assurance that anyone knew what the results would be. Some recognized they had an initial liking for certain functions of Canvas, but they also recognized that they did not know how those functions would affect learning experiences in the long run. Hesitancy to take ownership of a change or to take initiative for change occurred because people did not know what to expect for the future. For those who hesitated, the tendency then was to shift their focus from what they disliked to what they did like about the previous LMS because they were more comforted by what they knew for sure. The migration process tested the faith of all those involved. Each person affected by the migration faced the need to follow the process into the unknown.

\section{Limitations and Implications}

Although the data collected came from five key persons at one research institution, the findings from this study provide talking points and examples of what one academic institution has already experienced for academic institutions considering an LMS migration. In the consideration of these experiences, the data from the interviews have generated a theoretical sampling for the LMS migration process in which the sampling is based on concepts that are "deemed significant because (1) they are repeatedly present or notably absent when comparing incident after incident [in this case, each account made by the interviewees], and (2) through the coding procedures they earn the status of categories" (Strauss \& Corbin, 1990, p. 177).

From the theoretical sampling, and based on the Lewin's change management and ERP models, a proposed model emerged regarding LMS migration for this particular incident (see Figure 2). The core of decision-makers of LMS administration, the center for faculty support (including instructional designers), associate deans, and faculty members composed the search committee and became implementers of the migratory process. The decision of these key persons to move from Blackboard to Canvas coincided with the four main categories issuing from the data.

The factor of time stayed as a constant driving factor; the factors of the power of communication, the compatibility of technologies, and faith in the system were not constant and were lacking at times, thus represented by semi-permeable lines. As seen in the case, time constantly moved the migration forward, whereas any weakness with the latter factors hindered a smooth transition from one LMS to the other. For example, because the responsibility to communicate was not clearly designated to departments, there was the lack of communication from the decision-making implementers to the other implementers, thereby discouraging the other implementers to follow the recommended protocols to train and migrate to Canvas. However, when there was the presence of clear, strong testimonials of the migration process positively working, other implementers were more willing to adopt Canvas. 


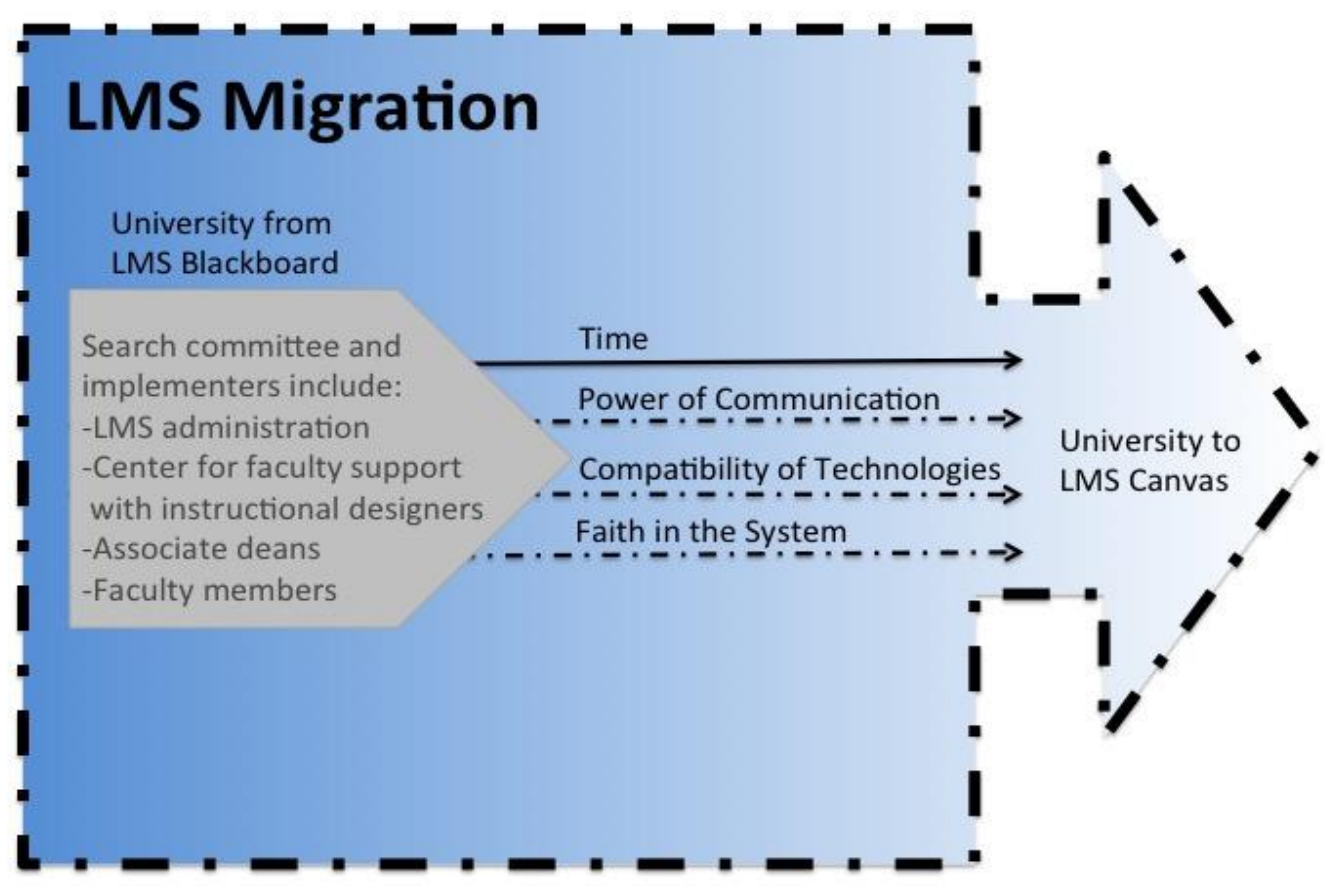

Figure 2. Theoretical sampling concerning LMS migration. The model above showcases how the decision of key members to change from one LMS to another LMS is realized through the constant component of time and the semi-permeable factors of the power of communication, the compatibility of technologies, and the faith in the system.

Finally, the LMS migration process itself, although considered its own system, has also been designated as semi-permeable and not closed off from possible external factors. The research team recognizes that other factors may have been involved in the process. Within the migratory process, the decision to migrate progressed along the four categories and eventually arrived at the actuality that the institute had fully integrated Canvas as the official LMS. Although that condition may have been the destination of the examined migratory process, as seen in regard to the faith in the system, the use of Canvas will continue to be a process. The future of the relationship between Canvas, and the institute will need further study.

\section{Conclusions}

As funding for academic institutions decreases and the need for academic institutions to become more like business enterprises increases, a massive change like an LMS migration will require more businesslike planning (Kremers \& Dissel, 2000). We recommend that more research be conducted on other cases of LMS adoption and integration and cases of subsequent future LMS migrations among institutes that 
have already migrated once. Additionally, more research might focus on the culture of academic institutions after they have integrated a new LMS.

Based on the findings from this study, diverse persons and multiple factors will contribute to the complexity of the entire LMS. As the literature suggests and is affirmed by this study, a crucial factor for successful LMS migration and LMS implementation is the involvement of faculty as primary stakeholders while ample communication among all stakeholders facilitates and eases the complex process of LMS transitions. Those vested with decision-making responsibilities will need to define and to consider what the short-term and long-term goals are with technology (Ryan et al., 2012). As more of the monolithic academic institutions that have prized their traditions integrate technology into their systems, although possibly begrudgingly, discussions about change related to pedagogy, curriculum development, institutional bureaucracy, and dissemination of information will become more frequent. In facilitating these discussions, the pitfall may be to focus on the technology (Sinkinson et al., 2014; Phelps \& Michea, 2003). The recommendation is that, more so now than ever, the administrators at academic institutions clearly articulate the vision for their institutions in order to use the technology effectively and to manage changes as seamlessly as possible.

\section{References}

Beatty, B., \& Ulasewicz, C. (2006). Faculty perspectives on moving from Blackboard to the Moodle learning management system. TechTrends, 50(4).

Chickering, A. W., \& Gamson, Z. F. (1987). Seven principles for good practice in undergraduate education. AAHE bulletin, 3,7 .

Chung, C. H., Pasquini, L. A., \& Koh, C. E. (2013). Web-based Learning Management System Considerations for Higher Education. Learning and Performance Quarterly, 1(4), 24-37.

Coates, H., James, R., \& Baldwin, G. (2005). A critical examination of the effects of learning management systems on university teaching and learning. Tertiary Education and Management, 11(1), 19-36. doi: 10.1080/13583883.2005.9967137

Ganesan, N. (2013). Migration of an e-learning model to the cloud. Journal of International Technology \& Information Management, 22(3), 19-36.

Harrington, T., Staffo, M., \& Wright, V. (2006). Faculty uses of and attitudes toward a course management system in improving instruction. Journal of Interactive Online Learning, 5(2), 178-190.

Hasselbring, W. (2000). Information system integration. Communications of the ACM, 43(6), 32-38.

Hudicka, J. (1998). An overview of data migration methodology. Retrieved from http://ww w.dulcian.com/articles/overview data migration methodology.htm

Jackson, G. A. (2014). Enterprise IT, e-learning, and transformation: prospects in higher education. Educause Review, 1-10.

SUCH, RITZHAUPT, \& THOMPSON / DOI: 10.5929/2017.7.2.6 
Jones, G. H., \& Jones, B. H. (2005). A comparison of teacher and student attitudes concerning use and effectiveness of web-based course management software. Educational technology \& society, 8(2), 125-135.

Kremers, M., \& Dissel, H. Van. (2000). Enterprise resource planning: ERP system migrations. Communications of the ACM, 43(4), 53-56.

Liaw, S.-S. (2008). Investigating students' perceived satisfaction, behavioral intention, and effectiveness of e-learning: A case study of the Blackboard system. Computers \& Education, 51(2), 864-873. doi: 10.1016/j.compedu.2007.09.005

Lonn, S., \& Teasley, S. D. (2009). Computers \& Education Saving time or innovating practice: Investigating perceptions and uses of Learning Management Systems. Computers \& Education, 53(3), 686-694. doi: 10.1016/j.compedu.2009.04.008

Machado, M., \& Tao, E. (2007, October). Blackboard vs. Moodle: Comparing user experience of learning management systems. Frontiers in Education Conference-Global Engineering: Knowledge Without Borders, Opportunities Without Passports, 2007. FIE'07. 37th Annual (pp. S4J-7). IEEE.

McGill, T., \& Klobas, J. (2009). A task-technology fit view of learning management system impact. Computers \& Education, 52(2), 496-508. doi: 10.1016/j.compedu.2008.10.002

Migration [Def. 1.1]. (2015). Oxford Dictionaries Online. Retrieved June 9, 2015, from http://www.oxforddictionaries.com/us/definition/american_english/migration.

Mohawk College. (2009). Final Report of the Learning Management System Project. Retrieved from http://Imsreview.mohawkcollege.ca/docstoshare/LMSreview FinalReport distribute.pdf

Oracle. (2011). Successful data migration. Retrieved from http://www.oracle.com/technetwork Lmiddleware/oedq/successful-data-migration-wp-1555708.pdf

Phelps, C., \& Michea, Y. (2003). Learning management systems' evaluation focuses on technology not learning. AMIA Annual Symposium Proceedings, 2003. Retrieved from http://www .ncbi.nlm.nih.gov/pmc/articles/PMC1480024/

Ray, J. A. (2009). An investigation of online course management systems in higher education: Platform selection, faculty training, and instructional quality. International Journal of Information and Communication Technology Education (IJICTE), 5(2), 46-59.

Ryan, T. G. T., Toye, M., Charron, K., \& Park, G. (2012). Learning management system migration: An analysis of stakeholder perspectives. The International Review of Research in Open and Distance Learning, 13(1), 220-237.

Sinkinson, C., Werner, M. J., \& Sieber, D. E. (2014). RAIT: A Balanced Approach to Evaluating Educational Technologies, 1-12. 
Smart, K., \& Meyer, K. (2005). Changing course management systems: Lessons learned. EDUCAUSE Quarterly, (2), 68-70. Retrieved from http://www.educause.edu/library/eqm05210

Sturgess, P., \& Nouwens, F. (2004). Evaluation of online learning management systems. Turkish Online Journal of Distance Education, 5(3), 1-9.

Sullivan, L. H. (1896). The tall office building artistically considered. Lippincott's Magazine, 403-409.

Syed, A. K., \& Raveendra, V. V. S. (2006). Data migration using iterative methodology. In IASTED International Conference Proceedings.

Turner, S. (2011). A self-study of technological transition: instructional impacts of shifting a distance course delivery system. Journal of Educators Online, 8(2), 1-24.

Zhu, Q. (2004). 2 critical stages for a successful ILS migration: system profiling and data conversion. Computers in Libraries, 24(3), 26-30.

\section{About the Authors}

Brenda L. R. Such, Ph.D. (brenlee@ufl.edu) earned her doctorate in Educational Technology within the College of Education at the University of Florida (UF) in Gainesville, Florida. Her research focuses primarily on active learning within STEM classes having blended learning formats. Such is presently an instructional designer and team leader for UF Center for Online Innovation and Production, which produces courses for UF Online and UF Distance and Continuing Education.

Albert D. Ritzhaupt, Ph.D. (aritzhaupt@coe.ufl.edu) is an associate professor at the University of Florida. His primary research areas focus on the design and development of technology-enhanced learning environments and technology integration in education. His publications have appeared in multiple venues, including the Journal of Research on Technology in Education, Computers in the Schools, Journal of Computing in Higher Education, Computers \& Education, Behavior Research Methods, Journal of Interactive Learning Research, Educational Technology Research and Development, Journal of Educational Computing Research, and Computers in Human Behavior.

George S. Thompson, Ed.S. (thompsongs2015@gmail.com) lives in Baton Rouge, Louisiana, and holds an Ed.S. from the University of Florida. His educational vita includes experiences as an instructor, technology coordinator, and educational technology and IT administrator in K-12 and community college education.

SUCH, RITZHAUPT, \& THOMPSON / DOI: 10.5929/2017.7.2.6 